\title{
A Inteligência Artificial e o Big Data no Setor da Saúde: Os Sistemas Especialistas e o Direito.
}

\author{
Artificial Intelligence and Big Data in the Health Sector: Expert \\ Systems and Law.
}

\author{
LUCA SCHIRRU
}

5211.luca@gmail.com/luca.schirru@pped.ie.ufrj.br

Advogado especializado em Direito da Propriedade Intelectual. Pós-Graduado em Direito da Propriedade Intelectual pela Pontifícia Universidade Católica do Rio de Janeiro. Mestre e Doutorando em Inovação, Propriedade Intelectual e Desenvolvimento (PPED- IE) pela Universidade Federal do Rio de Janeiro. Pesquisador do Núcleo de Estudos e Pesquisa em Direito, Artes e Políticas Culturais (NEDAC) e do Grupo de Estudos de Direito Autoral e Industrial (GEDAI).

RESUMO

O presente artigo terá como objetivo estudar os Sistemas Especialistas sob a perspectiva das novas tecnologias de Inteligência Artificial e Big Data e será organizado de maneira a, em um primeiro momento, apresentar os aspectos conceituais relativos à Inteligência Artificial e a Inteligência Artificial na Medicina (IAM), bem como apresentar os Sistemas Especialistas e relacionar tais sistemas com as novas tecnologias da Inteligência Artificial e Big Data. A segunda parte desse estudo irá propor algumas reflexões e problemáticas referentes aos Sistemas Especialistas sob a perspectiva do Direito, com maior foco em questões de Propriedade Intelectual, Responsabilidade, Privacidade e Ética. A terceira e última parte do estudo compreenderá as considerações finais sobre o tema e sugestões para estudos futuros.

Palavras-Chave: Inteligência Artificial na Medicina. Big Data. Sistemas Especialistas. Propriedade Intelectual.

ABSTRACT

This work aims to study the Expert Systems under the perspective of the new technologies of Artificial Intelligence and Big Data and will be organized as follows: at first will be presented the conceptual aspects of Artificial Intelligence, Artificial Intelligence in Medicine (IAM) and the expert systems, which will be analysed in accordance with the aforementioned technologies. The second part of this study will propose some thoughts and present relevant issues related to expert systems from the perspective of law, with greater focus on intellectual property matters as well as liability, privacy and ethics issues. The third and final part of the study will present the final considerations on the subject matter and suggestions for future studies.

Keywords: Artificial Intelligence in Medicine. Big Data. Expert Systems. Intellectual Property. 


\section{INTRODUÇÃO}

A IBM, conhecida pela fabricação de PCs e hodiernamente pela sua grande atuação no setor de serviços relacionados à software é também a detentora da tecnologia denominada Watson. Watson é um sistema baseado em Inteligência Artificial que foi capaz de vencer o programa Jeopardy!, onde o participante é exposto a uma determinada resposta e deve adivinhar qual foi a pergunta realizada. Para o presente trabalho, o que importa não é o desempenho de Watson no Jeopardy, e sim a sua nova identidade e natureza: Dr. Watson, um Sistema Especialista.

A IBM, através da aquisição de uma empresa que possui cerca de trinta bilhões de imagens (Raios-X, Scans de Ressonância Magnética e tomografia computadorizada), está trabalhando na inserção de tecnologias de Inteligência Artificial no setor de diagnósticos médicos. ${ }^{1}$ Assim, essas imagens seriam utilizadas para "treinar" Watson, uma tecnologia de Inteligência Artificial, no reconhecimento de sinais de câncer e patologias cardíacas através de técnicas conhecidas como “deep learning”, que permitem o reconhecimento de padrões através da análise de uma imensidão de dados ${ }^{2}$.

Importante notar que existe um cuidado por parte daqueles que trabalham na evolução e aprimoramento do Watson em manter essa tecnologia como uma tecnologia de assistência ao médico e não de substituição, mesmo que ele passe um dia a ter uma base de conhecimento muito superior a um médico humano e tenha capacidade de promover diagnósticos mais confiáveis em termos técnicos ${ }^{3}$.

O caso de Watson foi escolhido para introduzir o presente estudo, pois tal caso, em suas especificidades, ilustra bem a estrutura e o conteúdo do presente artigo. Em primeiro lugar, destaca-se de pronto que o presente estudo terá como objetivo estudar os Sistemas Especialistas, sob a perspectiva das novas tecnologias de Inteligência Artificial e Big Data.

Ainda, o uso de imagens e dados por um Sistema Especialista não chama atenção apenas pela questão do Big Data, mas também por aspectos de Propriedade Intelectual de Base de Dados e Privacidade de informações.

\footnotetext{
${ }^{1}$ Mcmillan; Dwoskin, 2015.

${ }^{2}$ Mcmillan; Dwoskin, 2015

${ }^{3}$ Brynjolfsson; Mcafee, 2014
} 
A Inteligência Artificial e o Big Data no Setor da Saúde: Os Sistemas Especialistas e o Direito.

Por fim, tal como foi destacado acima e mesmo que de maneira mais ampla, serão discutidas algumas questões de caráter ético acerca da aplicação de tecnologias de inteligência artificial na área da saúde.

O presente trabalho será organizado de maneira a, em um primeiro momento, apresentar os aspectos conceituais relativos à Inteligência Artificial e a Inteligência Artificial na Medicina (IAM), bem como apresentar os Sistemas Especialistas e relacionar tais sistemas com as novas tecnologias da Inteligência Artificial e Big Data. A segunda parte desse estudo irá propor algumas reflexões e problemáticas referentes aos Sistemas Especialistas sob a perspectiva do Direito e com foco em questões de Propriedade Intelectual, Privacidade e Ética, seguido de considerações finais sobre o tema.

\section{INTELIGÊNCIA ARTIFICIAL E SAÚDE}

\subsection{INTELIGÊNCIA ARTIFICIAL}

Alan Turing ${ }^{4}$, em seu trabalho intitulado Computing Machinery and Intelligence, propôs a seguinte pergunta: "podem as máquinas pensar?"5. Ainda, Turing finaliza seu artigo afirmando que se espera que as máquinas venham a competir com os seres humanos em todos os campos intelectuais ${ }^{6}$. Tais questionamentos remontam a década de 50 e hoje ressoam cada vez mais alto dentre os estudiosos da ciência e, consequentemente, do Direito.

De acordo com Russell e Norvig (2010), o estudo da inteligência em si é algo que filósofos buscam há cerca de dois mil anos, portanto, definir a Inteligência Artificial não será uma tarefa capaz de ser exaurida em poucos parágrafos. Destacam esses autores que acerca da concepção do que vem a ser inteligência artificial existem quatro grupos de abordagens: (i) sistemas que Pensam como Humanos ${ }^{7}$; (ii) sistemas que

\footnotetext{
${ }^{4}$ Turing, Alan M. Computing Machinery and Intelligence. Mind. Vol. 49. 1950. Pp. 433-460. p. 433.

${ }^{5}$ Russel e Norvig (2010) bem destacam que Turing, mesmo realizando a pergunta acima, afirmou que a pergunta certa seria se uma máquina seria capaz de passar em um teste comportamental desenvolvido por ele.

${ }^{6}$ Turing, 1950. p.460

${ }^{7}$ De acordo com os estudos de Russel e Norvig (2010), o item (i) compreende os sistemas que pensam como humanos e está baseado em uma ciência congnitiva, onde se busca, através de experimentos baseados na psicologia e estudos de IA, desenvolver teorias capazes de explicar o funcionamento da mente humana, partindo do pressuposto de que assim que for possível ter uma teoria sobre a mente humana, será possível estudar o funcionamento do pensamento artificial desenvolvido por um programa de computador.
} 
pensam racionalmente ${ }^{8}$; (iii) sistemas que agem como humanos ${ }^{9}$ e (iv) sistemas que agem racionalmente ${ }^{1011}$. Tais abordagens, como bem destacado pelos autores acima, podem ser divididas em duas dimensões maiores: uma dimensão focada no comportamento (iii e iv) e uma dimensão focada no processo cognitivo (i e ii) ${ }^{12}$.

Se valendo do trabalho de Russel e Norvig (2010), Hengstler, Enkel e Duelli (2016) destacam que existem dois grandes grupos de IA, uma IA forte e uma IA fraca. A IA forte seria baseada em tecnologias ainda não existentes e consistiria em um sistema dotado de inteligência humana ou até mesmo superinteligência em todos os aspectos. Por outro lado, a IA fraca seria aquela verificada nas tecnologias atuais e consistiria no fato de que a IA seria capaz de executar tarefas limitadas a determinadas capacidades humanas, como lógica etc, e que são de interesse para aplicação comercial. Na mesma esteira de Brynjolfsson e McAfee (2014), poderia se dizer que a IA fraca não englobaria aspectos que ainda são particulares do ser humano, como a capacidade de "pensar fora da caixa", de inovar, de idealizar, de criar algo.

Dessa maneira, não obstante o termo "Inteligência Artificial" instigar nas pessoas o sentimento de algo digno de ficção-científica, o presente estudo se prestará a estudar tecnologias de IA "Fraca", que já são realidade nos mais diversos campos de

\footnotetext{
${ }^{8} \mathrm{O}$ item (ii) refere-se aos sistemas que pensam racionalmente e também é conhecimento como a abordagem das leis do pensamento e que fundamentaram o campo de pesquisa da lógica, de grande uso e influência nas tecnologias de inteligência artificial (RUSSEL, NORVIG, 2010).

${ }^{9}$ De acordo com os estudos de Russel e Norvig (2010), no que se refere ao item (iii), os sistemas que agem como humanos podem ser relacionados com o Teste de Turing, onde tal cientista entende que o comportamento inteligente estaria relacionado à capacidade de se realizar tarefas cognitivas como um ser humano. Ou seja, passaria no teste de Turing uma máquina dotada de determinadas capacidades como comunicação, armazenamento e utilização de informações armazenadas durante uma entrevista e aprendizado capaz de se adaptar a determinadas circunstâncias. Uma máquina que fosse dotada de tais capacidades e que fosse capaz de realizar tais tarefas de maneira humana até mesmo a enganar um entrevistado, passaria no teste de Turing.

10 A abordagem do agente racional, ou seja, os sistemas que agem racionalmente são aqueles referenciados no item (iv). Tal abordagem possui relação com a abordagem logicista, haja vista que para um agente agir racionalmente, este deve, de certa maneira pensar racionalmente, mas não é apenas isso, haja vista que determinadas ações não possuem certo ou errado e outras não são passíveis de ser pensadas previamente (RUSSEL, NORVIG, 2010).

${ }^{11}$ Russel e Norvig (2010, p.7-8) se valem de tal abordagem em sua obra sob os argumentos de que este possui algumas vantagens sobre os demais, como segue: The study of AI as rational agent design therefore has two advantages. First, it is more general than the "laws of thought" approach, because correct inference is only a useful mechanism for achieving rationality, and not necessary one. Second, it is more amenable to scientific development than approaches based on human behavior or human thought, because the standard of rationality is clearly deifined and completely general. Human behavior, on he other hand, is well-adapted for one specific environment and is the product, in part, of a complicated and largely unknown evolutionary process that still may be far from achieving perfection.

${ }^{12}$ Russel e Norvig, 2010.
} 
A Inteligência Artificial e o Big Data no Setor da Saúde: Os Sistemas Especialistas e o Direito.

estudo e cuja expansão certamente causará questionamentos e problemáticas no campo do Direito, que merecem ser comentadas.

\subsection{INTELIGÊNCIA ARTIFICIAL E SAÚDE: A INTELIGÊNCIA ARTIFICIAL NA MEDICINA (IAM)}

No que se refere à relação entre a inteligência artificial e a área da saúde, chamase atenção para um campo de pesquisa denominado Inteligência Artificial na Medicina, que consiste na aplicação de tecnologias de inteligência artificial na Medicina. Essas tecnologias podem ser das mais diversas aplicações, desde softwares especialistas capazes de prover diagnósticos, computação bioinspirada mediante a utilização de redes neurais artificiais ${ }^{13}$, até robôs que auxiliam cirurgiões em operações de alta precisão.

Algumas dentre as mais recentes tecnologias são capazes de permitir a pessoas com limitações na visão que vivenciem alguns aspectos relacionados a esse sentido ${ }^{14}$, cadeiras de rodas com possibilidade de controle pela mente e até mesmo computadores usados para outros fins, como o Watson da IBM, estão sendo utilizados para fins médicos através do estudo de todo o material médico de alta qualidade já publicado para auxiliar os profissionais dessa área em diagnósticos ${ }^{15}$. Outras aplicações de sistemas de inteligência artificial na saúde que serão relevantes para a discussão aqui proposta são aquelas referentes aos cuidadores dotados de inteligência artificial, que podem atuar através do aconselhamento e atendimento clínico de pacientes, de maneira mobile inclusive ${ }^{16}$.

A doutrina sobre Inteligência Artificial na Medicina relaciona a evolução das aplicações das tecnologias de IA em quatro estágios. Cumpre notar que o estudo de referência para essa evolução histórica data de 1997, tendo sido desenvolvidas tecnologias mais avançadas nos últimos vinte anos. Entretanto, o que se busca

\footnotetext{
${ }^{13}$ Em Guarizi, Oliveira (2014, p. 32): “As RNAs podem ser aplicadas na área médica sendo conhecidas como Computação Bioinspirada. Esta é a área de pesquisa da computação que estuda e desenvolve técnicas computacionais inspiradas na biologia resolvendo problemas práticos. Problemas este como o DNA, para obter-se os resultados de um material genético, o computador agrupa dados biológicos e os classifica-os. Além dos testes poderem fazer o reconhecimento de paternidade, pode-se realizar comparação entre espécies de animais. Outra aplicação na medicina é o descobrimento de determinadas patologias através do DNA, podendo até mesmo descobrir as chances que o paciente desenvolver determinada doença e os tipos de problemas. “

${ }^{14} \mathrm{http} / / / \mathrm{www} .0$ rcam.com/

${ }^{15}$ Brynjolfsson e McAfee, 2014.

${ }^{16}$ Luxton, 2014.
} 
identificar é a partir de onde se deu a introdução de técnicas de IA mais complexas, com a utilização de Redes Neurais Artificiais, por exemplo. Para tanto, valemo-nos da figura desenvolvida por Fernandes (1997, p. 36) e adaptado para o presente trabalho:

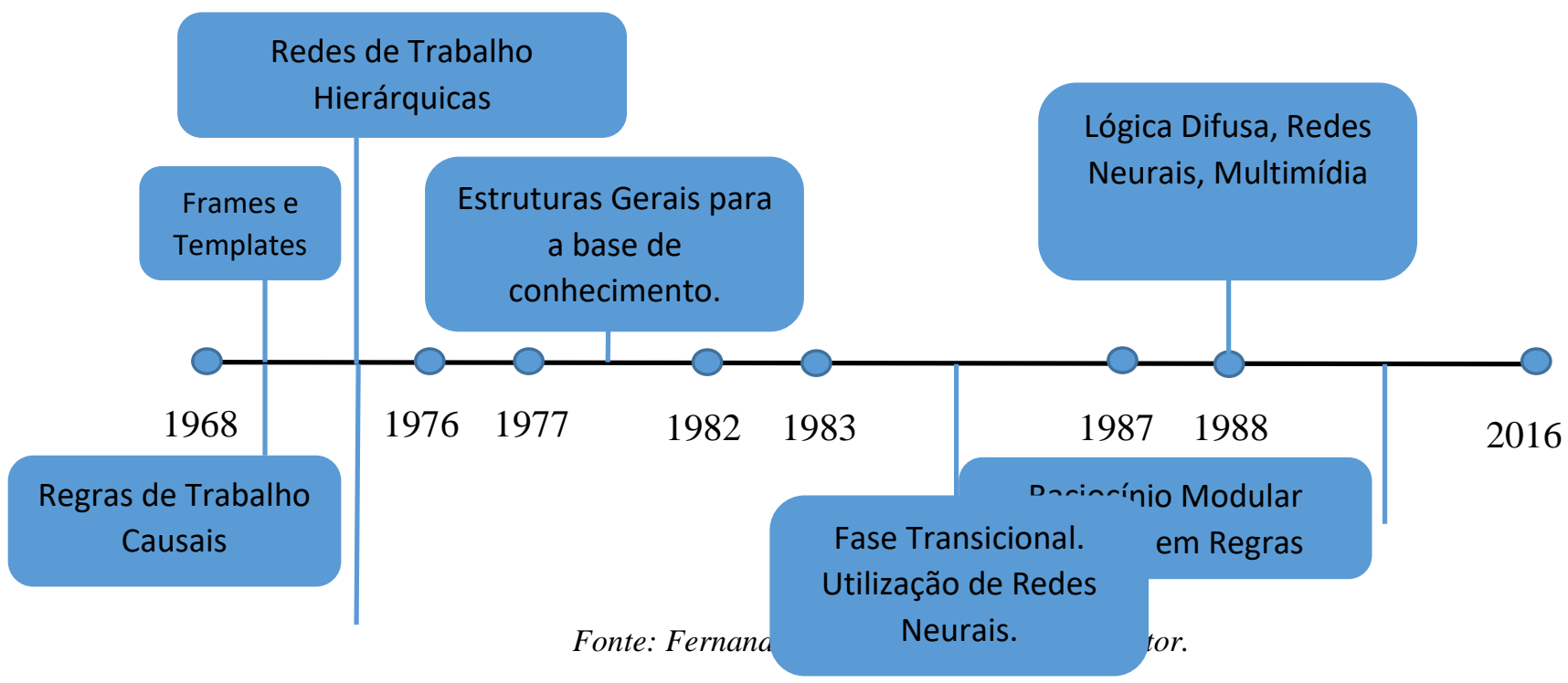

2.2.1. Os Sistemas Especialistas

Uma das aplicações mais recorrentes da Inteligência Artificial na Medicina é o uso de Sistemas Especialistas que, segundo Guarizi e Oliveira (2014, p. 27) "são programas planejados para adquirir e disponibilizar o conhecimento operacional de um especialista humano em uma determinada área ou campo de atuação.”. Em suma, seriam, como bem define Westphal (2003, p. 26): "Uma aplicação da Inteligência Artificial que atua em uma determinada área de conhecimento.”.

Tais sistemas são utilizados em situações de tomada de decisão nas atividades médicas, notadamente em diagnósticos, prognósticos e planejamento terapêutico ${ }^{17}$, sendo a sua utilidade tanto para dar suporte à decisão a ser tomada pelo médico especialista ou o próprio sistema tomar a decisão ${ }^{18}$.

Vale acrescentar que tais sistemas também se valem de tecnologias de Redes Neurais Artificiais, principalmente no caso de Sistemas de Apoio a Decisão Clínica (SADM), que são "softwares desenvolvimentos para aprenderem com base nos dados

\footnotetext{
${ }^{17}$ Guarizi, Oliveira, 2014, p.32.

${ }^{18}$ Widman, 1995.
} 
A Inteligência Artificial e o Big Data no Setor da Saúde: Os Sistemas Especialistas e o Direito.

inseridos e ajudarem na análise de dados como imagens de mamografias, colonoscopias ou mesmo dados de acompanhamento clínico para prognósticos"19.

Segundo Widman (1995) a composição de um sistema especialista pode ser visualizada na ilustração abaixo:

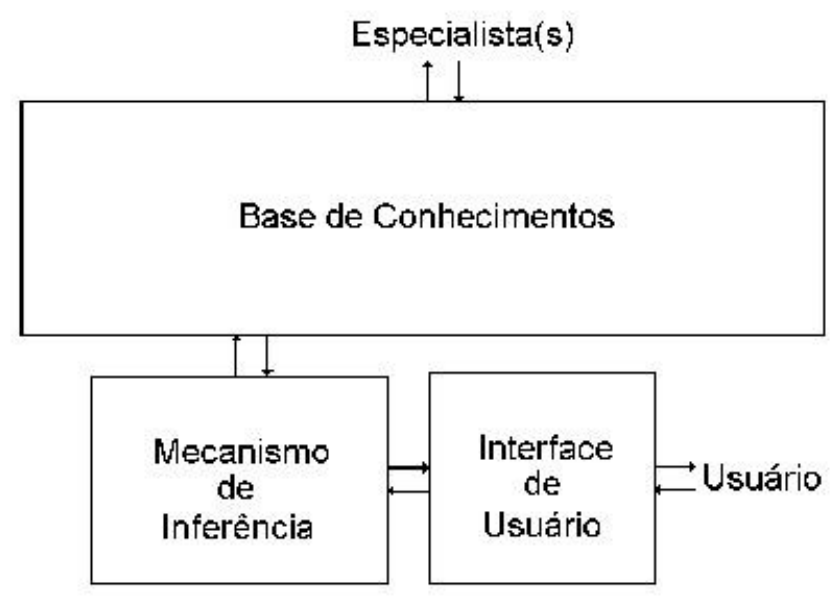

Fonte: Widman (1995)

Para o presente trabalho, no que se refere à imagem acima importam as noções de base de conhecimento e mecanismo de inferência. A base de conhecimento, segundo Westphal (2003, p. 27),

representa o conhecimento do "domínio" do problema, extraído de um especialista humano, e o motor de inferência constitui-se no modo com o qual se vai processar as informações da base, para chegar à conclusão adequada. A eficácia do SE estará condicionada a uma base constituída de todo o conhecimento necessário.

Relacionado com os fenômenos tecnológicos mais recentes, importa o estudo das Bases de Conhecimento dada a relevância dos debates acerca do Big Data nas discussões principalmente sobre privacidade. Importa, portanto, a análise de tais dados de acordo não só com a privacidade, mas também a sua relação com o mecanismo de inferência.

${ }^{19}$ Guarizi, Oliveira, 2014, p.32. 
O mecanismo de inferência, que se constitui por um algoritmo "capaz de elaborar as conclusões a partir dos dados fornecidos pelo usuário do sistema, e do conhecimento armazenado em suas bases." ${ }^{20}$, pode ser relacionado com as tecnologias de Inteligência Artificial e é exatamente através da diferença entre a forma como a base de conhecimento é estruturada e como se dá o funcionamento do mecanismo de inferência que podem se classificar os diferentes tipos de Sistemas Especialistas ${ }^{21}$.

As complexidades relacionadas aos Sistemas Especialistas sob a perspectiva do Direito da Propriedade Intelectual serão aprofundadas no item abaixo.

\section{REFLEXÕES SOBRE A APLICAÇÃ̃ DA INTELIGÊNCIA ARTIFICIAL E DO BIG DATA NA SAÚDE: PRIVACIDADE, ÉTICA, RESPONSABILIDADE E PROPRIEDADE INTELECTUAL.}

\subsection{PRIVACIDADE}

O crescimento do Big Data impactou também no setor da saúde, principalmente nas pesquisas envolvendo técnicas de datamining e outras técnicas capazes de incorporar diversas fontes de dados existentes. Entretanto, antes de comentar as questões de privacidade relacionadas ao Big Data na área médica, cumpre apresentar, mesmo que de forma sucinta, o que é o Big Data.

O que se pode notar já em um primeiro momento é que a própria conceituação do que vem a ser o Big Data não recebe tratamento pacificado pela doutrina ${ }^{22}$, ou seja, sequer a tecnologia em questão possui um conceito definido dentre os estudiosos dessa tecnologia não só na área jurídica, como também no campo da economia e da informática. Entretanto, para fins de uma melhor contextualização do tema, cumpre destacar abaixo o conceito de Big Data atribuído por Albagli, Appel e Maciel (2013, p.4), por ser bem esclarecedor:

Big Data compreende a geração, tratamento e análise de grandes volumes de dados que excedem as capacidades convencionais de processamento, e vem sendo explorado também por empresas, governos e outros segmentos interessados em extrair informações a partir de grandes quantidades de dados não estruturados.

\footnotetext{
${ }^{20}$ Widman, 1995.

${ }^{21}$ Widman, 1995.

22 Carloni, 2013; Gonçalves, 2014; Breternitz, Silva, 2013; Kemp, 2014.
} 
Retornando à discussão acerca desse tema na área da IAM, de acordo com Yang e Veltri (2015, p.2), as fontes de dados utilizadas no setor de saúde consistem em: (i) registros médicos eletrônicos e textos clínicos; (ii) sinais e imagens biomédicas; (iii) dados provenientes de sensores utilizados nesse setor; (iv) base de dados genômicas e farmacêuticas; (v) sistemas de relatórios espontâneos; (vi) literatura de saúde e biomedicina e (vii) dados de mídias sociais.

Enquanto determinadas fontes de dados não levantam tantos questionamentos acerca da possibilidade de uso de tais informações sem prejuízo à privacidade ${ }^{23}$ do paciente, como seria o caso de dados obtidos a partir de artigos científicos e imagens não identificadas ou identificáveis, a questão sobre dados como aqueles provenientes de mídias sociais e registros médicos não é de fácil resolução.

Existem, inclusive, métodos indicados pela legislação norte-americana, por exemplo, para que os pacientes sejam desassociados de seus relatórios, o que Berman (2002) relaciona a viabilidade com a aplicação de determinados algoritmos relacionados à disciplina de Inteligência Artificial através de algoritmos de "anonymization" 24 e "deidentification" 25 .

${ }^{23}$ Uma das importantes contribuições do estudo de Berman (2002) sobre questões relacionadas à confidencialidade em práticas de data mining é a diferença entre Confidencialidade, Privacidade e Segurança no tratamento de informações relacionadas à pacientes ou a um determinado tratamento. Segundo Berman (2002, p.27), a Confidencialidade estaria violada quando um pesquisador ou aquele que detém a confiança do paciente revela ou transmite uma informação para um terceiro desautorizado concernente aquele paciente sem a sua permissão prévia. Por outro lado, destaca Berman (2002, p.27) que a Privacidade estaria relacionada a invasão na vida privada de um paciente exposto a um estudo ou pesquisa, por exemplo e, por fim, a Segurança estaria relacionada ao acesso de um indivíduo desautorizado aos registros do paciente.

${ }^{24}$ Em Berman, 2002, p. 30: "An anonymization algorithm removes all patient identifiers in a record. Most hospital information systems have a well-defined set of patient identifiers (name, social security number, medical record, etc.). These usually are accessed from reserved data dictionary fields. Once these reserved fields are accessed, all other fields (including free text fields) can be parsed and deleted of any identifier matches. So, if the patient's name (as found in the "name" field) is Thomas Patterson, then any mention of Thomas Patterson should be deleted from free-text patient fields (such as admission notes, history notes, discharge notes). In addition, a robust algorithm should search and destroy any reference to Tom Patterson, or just plain Tom or the word Mr., Miss, Mrs., or Ms. followed by any name. It may be beneficial to have a list of all the names of patients registered in the hospital system and delete any freetext match (even if the name is not the name of the person attached to the record). It might be useful to remove all mention of staff doctor names or of any surname following the Dr. title. A similar process might be applied to the medical record number or social security number and would include all variations of presentation of a social security number (with or without hyphenation)."

${ }^{25} \mathrm{Em}$ Berman, 2002, p. 31: "One of the keystones of deidentification process is the creation of datasets that contain no unique records (with the exception of the unique identifier code). If every record has at least one additional record to which it is identical, then it becomes logically impossible to distinguish any one individual's record from any of the other individuals whose records contain the same data elements. 
Ainda, a respeito da possibilidade de uso de tais dados de maneira anônima se posiciona Berman (2002, p. 34):

There is no legal or ethical reason to withhold anonymized datasets from the public. Quite the opposite. Anonymized datsets have enormous value to other researchers who can merge your data with theirs, derive new ways of analyzing your data, or develop new questions that can be addressed by your dataset. Researchers who have created anonymized datasets should seriously consider publishing their data as a primary resource or as a secondary resource attached to any publication that results from the research project. (...) Issues of intellectual property impacting on the investigator and the institution (e.g. ownership, licensing of data, derivative work "reachthrough") have accumulated very little legal precedent.

Como abordado em Berman (2002), Vacca (2013) aponta para a existência de leis que protegem determinados tipos de dados médicos como a HIPPA (Health Insurance Portability and Accountability Act of 1996) e a GINA (Genetic Information Nondiscrimination Act of 2008) e questiona a proteção desses dados pessoais relacionados a saúde, inclusive a existência de um aumento nos erros baseados em informações não-identificáveis, bem como a possibilidade de tais leis imporem obstáculos ao exercício da medicina através de ferramentas digitais.

Ainda no que se refere ao uso de dados, Vacca (2013) destaca que alguns pacientes não estão preocupados com o uso de dados relacionados à sua condição de saúde, haja vista que não possuem qualquer condição capaz de os sujeitar à exposição, preconceito etc.

Entretanto, a questão conceitual acerca da privacidade no meio médico e a forma como se lidar com isso ainda é objeto de problemas, pois mesmo que se decida que a regra é o compartilhamento, devendo aqueles que não desejam expor suas informações informarem desde já, tal ato já levantará suspeita acerca de sua situação, podendo, inclusive, o expor da mesma forma ${ }^{26}$.

\subsection{QUESTÕES ÉTICAS}

Luxton (2014, p.7) aponta que a extensão nas quais tecnologias de inteligência artificial podem ser aplicadas na área médica dependerá de um equilíbrio entre seus prós

Guaranteeing that any medical dataset contains only ambiguous records (i.e. records with multiple identical instances) is a feasible computational task. It may involve constantly revising the scope of certain data elements (such as using only the first few digits of zip codes, or using only the State to mark a patient's address) or even adding fake (ambiguating) records to the data. The method of deidentification would depend largely on the purpose of the data mining effort."

${ }^{26}$ Vacca. 2013. 
A Inteligência Artificial e o Big Data no Setor da Saúde: Os Sistemas Especialistas e o Direito.

e contras. De um lado, esses sistemas trazem alguns benefícios para os usuários a partir do momento que podem ser acessados através de tecnologias mobile, bem como tais tecnologias possui capacidade de aprendizado, acesso a uma vastidão de dados e capacidade de integração de dados de diferentes fontes, o que o permite fornecer tratamentos mais apropriados para cada caso.

Além disso, entende Luxton (2014, p. 7) que outra vantagem desses sistemas é o fato de que estes não estão sujeitos à falha humana: erros cognitivos, esquecimento, cansaço, tédio, stress, preconceitos, ansiedade etc, e, portanto seriam capazes de prover o tratamento mais personalizado para o paciente. Do ponto de vista econômico também seu uso pode ser benéfico, ao aumentar a produtividade e eficiência da prestação de determinados serviços.

Por outro lado, há que se levantar também questionamentos sobre a adoção de tais tecnologias em determinados campos da medicina, como seria no caso da saúde mental, onde a empatia entre o terapeuta e o paciente é fator fundamental para o processo de tratamento onde é comum que os pacientes se sintam melhor de ver que são compreendidos pelo seu terapeuta, que também é humano e compartilha dos seus sentimentos ${ }^{27}$. Além disso, casos como os de tendência suicidas são amenizados através do envio de mensagens de cuidado pelo terapeuta ou cuidador, reforçando a questão da empatia, o que não seria necessariamente verdade com uma máquina, pois a máquina não se preocuparia legitimamente com aquele ser humano ${ }^{28}$.

Luxton (2014, p. 8) explicita a importância da relação interpessoal na psicoterapia como verificado abaixo:

The psychotherapeutic process typically involves mutual discovery, a collaborative breaking down of the issues at play, the sharing of inner most thoughts, feelings, desires, and mutual discussion of the therapeutic relationship and process. The human psychotherapist is not perfect; she/he learns from the patient and the patient knows that the psychotherapist has limitations and sometimes makes mistakes because they are human. The imperfection of the psychotherapist is, in fact, essential to the psychotherapeutic process and psychotherapy with an AICP may not be optimal if the AICP is perceived as knowing everything or is "too perfect".

\subsection{PROPRIEDADE INTELECTUAL}

\footnotetext{
${ }^{27}$ Luxton, 2014.
}

${ }^{28}$ Luxton, 2014. 
No que se refere à Propriedade Intelectual, a questão que será ressaltada no presente estudo é o tratamento concedido às bases de dados, como as utilizadas pelos Sistemas Especialistas, pelo Direito Autoral, bem como a questão autoral quando envolver sistemas de Inteligência Artificial.

Segundo Marcos Wachowicz ${ }^{29 ، A}$ base de dados sendo um conjunto de informações referentes a um determinado setor do conhecimento humano, está organizada por meio de programas de computador especialmente desenvolvidos para esta finalidade". Com o aumento do fluxo de dados das mais diversas fontes, tecnologias de Inteligência Artificial vem sendo utilizadas para identificar padrões, como seria o caso de um software que identificaria padrões em imagens médicas com o objetivo de apontar tumores.

Nesse sentido, complementam Breternitz e Silva (2013, p.112):

\begin{abstract}
Em adição ao próprio avanço tecnológico que o streaming computing proporciona, diferentes técnicas de Inteligência Artificial são empregadas para tratar dados em diferentes estruturas e descobrir através desses, padrões em menor tempo e com maior precisão. Para dar suporte a tudo isso, novos paradigmas de banco de dados vem surgindo para trabalhar com alto volume de dados e em tempo real.
\end{abstract}

A proteção às bases de dados no Brasil não é sobre o seu conteúdo, mas sim sobre a sua organização, seleção ou disposição do seu conteúdo ${ }^{30}$. Como agir, então, perante a uma base de dados organizada e estrutura por um sistema de inteligência artificial?

$\mathrm{O}$ art. 11 da LDA determina que o Autor "é a pessoa física criadora de obra literária, artística ou científica" e que dentro de determinadas hipóteses previstas na Lei a proteção concedida ao autor também poderia ser aplicada às pessoas jurídicas, tal como a titularidade. Dessa maneira, deixa claro que o autor de uma determinada obra deverá ser uma pessoa física.

Some-se a esse entendimento o disposto do art. $7^{\circ}$ da LDA sob o qual são passíveis de proteção sob o Direito Autoral “criações de espírito, expressas por qualquer

\footnotetext{
${ }^{29}$ WACHOWICZ, Marcos. A Proteção Jurídica Das Bases De Dados Em Face Da Revolução Da Tecnologia Da Informação. http://www.gedai.com.br/sites/default/files/arquivos/artigo-base-dados-marcos-wachowicz.pdf.

30 Art $7^{\circ}$, XIII da Lei ${ }^{\circ}$ 9.610/98: "Art. $7^{\circ}$ São obras intelectuais protegidas as criações do espírito, expressas por qualquer meio ou fixadas em qualquer suporte, tangível ou intangível, conhecido ou que se invente no futuro, tais como: (...)XIII - as coletâneas ou compilações, antologias, enciclopédias, dicionários, bases de dados e outras obras, que, por sua seleção, organização ou disposição de seu conteúdo, constituam uma criação intelectual."
} 
ISSN ELETRÔNICO 2316-8080

A Inteligência Artificial e o Big Data no Setor da Saúde: Os Sistemas Especialistas e o Direito.

meio ou fixadas em qualquer suporte, tangível ou intangível, conhecido ou que se invente no futuro" 31 .

No que se refere especificamente ao art. $7^{\circ}$ da LDA, chama atenção o termo "criações do espírito" utilizado, o que condicionaria a proteção de uma obra intelectual a uma relação com um agente dotado de um espírito. Ou seja, não basta que o responsável por aquela obra seja dotado de inteligência, é necessário que aquela obra seja uma criação do espírito daquele autor.

Entretanto, referente à essa questão polêmica, que levantaria até debates de natureza teológica, preferimos entender no mesmo sentido de Davies (2015) de que não cabe a lei dispor sobre questões religiosas, principalmente no Brasil, um país Laico. Portanto, entendemos que o termo 'criação do espírito' não deva ser levantado no sentido metafísico/espiritual para definir se uma determinada obra pode ser considerada ou não como passível de proteção autoral, mas sim como um animus de criar.

Dessa maneira, e partindo da premissa que a regra sob a legislação nacional atual é de que o autor seria apenas pessoa física, estaria uma obra desenvolvida por um sistema de inteligência artificial em domínio público em um momento imediato a sua criação? Pertenceria ao programador daquele programa? Pertenceria à empresa titular daquele programa, através de uma interpretação analógica do art. $4^{\circ}$ da Lei no 9.609/98?

Pode ocorrer de as perguntas acima levarem a uma questão mais complexa: estaria a legislação nacional atual apta a lidar com questões envolvendo agentes de inteligência artificial ou é necessário um novo aparato legal mais atualizado?

A questão é complexa e demanda estudos que aprofundariam a discussão ao nível do estudo de conceitos fundamentais do direito e, principalmente, do direito autoral.

\subsection{RESPONSABILIDADE}

Outra questão que se faz pertinente ao debater a questão da Inteligência Artificial, não só na medicina, mas em todos os campos, é a questão da responsabilidade. Como já foi visto no presente estudo, determinados sistemas dotados de inteligência artificial são capazes de prover diagnósticos precisos e até mesmo um

\footnotetext{
${ }^{31}$ Lei ${ }^{\circ}$ 9.610/98. Art. $7^{\circ}$ São obras intelectuais protegidas as criações do espírito, expressas por qualquer meio ou fixadas em qualquer suporte, tangível ou intangível, conhecido ou que se invente no futuro.
} 
planejamento terapêutico personalizado. Mas como seria o caso de um sistema que cometesse um erro capaz de prejudicar um determinado indivíduo? Quem seria responsável pelos danos causados?

Ainda, os danos causados não necessariamente seriam consequências de um erro, mas também poderiam ser consequência de escolhas. Ora, a função de um sistema de Inteligência Artificial é a persecução de um determinado objetivo, que pode ser alcançado mediante o aprendizado e as decisões tomadas por esse agente no decorrer das suas experiências. Isso gera consequências relevantes para o Direito: dentre elas o fato de que as atitudes e decisões de um agente de inteligência artificial passam a ser imprevisíveis ao ser humano ${ }^{32}$. Dessa maneira, uma decisão tomada por um sistema de inteligência artificial pode ser a matematicamente mais apropriada, mas não a "humanamente" mais apropriada. Esse é um ponto importante no que se refere à autonomia desses sistemas em situações sensíveis, como é o caso da medicina, onde o elemento humano é necessário, mesmo com toda a precisão técnica e científica desses sistemas.

Na legislação brasileira, especificamente no Código Civil, a questão da responsabilidade civil pode ser verificada nos arts. 931 e 932 que dispõem que, ressalvados casos específicos, as empresas possuem responsabilidade objetiva pelos danos causados pelos seus produtos postos em circulação, bem como também são responsáveis objetivamente pelos danos causados pelos seus empregados e prepostos no exercício do seu trabalho.

Nesse ponto, traçando um paralelo com a legislação autoral, se for realizada uma interpretação analógica do Art. $4^{\circ}$ da Lei $n^{\circ} 9.609 / 98$ no sentido que pertenceria ao empregador ou à empresa o produto desenvolvido por um programa de computador ou robô dotado de inteligência artificial, também seriam responsáveis esses agentes de maneira objetiva quando do cometimento de ato ilícito por tal sistema de inteligência artificial.

\footnotetext{
${ }^{32}$ Conforme bem apontam os autores, com o crescimento da capacidades dos computadores, o seu autotreinamento, potencial de treinamento e capacidade criativa, a diferença entre o desempenho de um cérebro humano e o desempenho de uma máquina dotada de um sistema de Inteligência artificial tende a reduzir cada vez mais, chegando a um ponto, em um futuro não muito distante, onde a capacidade de desempenho e funcionamento de um agente de inteligência artificial pode até mesmo superior a capacidade de um cérebro humano (ČERKAA; GRIGIENĖA; SIRBIKYTĖB, 2015 p. 382).
} 
Entretanto, a questão não é tão simples, haja vista que, antes de se definir questões de responsabilidade e autoria de um sistema de inteligência artificial, é necessário compreender a sua posição dentro do ordenamento jurídico vigente.

A quem deveria ser atribuída a responsabilidade então nos casos onde fosse licenciado um programa de computador dotado de Inteligência Artificial, capaz de alterar seu código através do aprendizado e de tomar decisões com bases no seu aprendizado e na disponibilização de informação em uma rede mundial de informações geradas por outros sistemas de inteligência $\operatorname{artificial}^{33}$ ?

\section{CONCLUSÃO}

Como se pôde observar no presente estudo, as aplicações de sistemas de Inteligência Artificial e Big Data na Medicina são diversas, bem como as questões polemicas envolvidas em cada aplicação, seja sob a ótica do Direito, da Ética etc. Ainda, dado o recente surgimento e desenvolvimento de tais tecnologias, não foram identificados muitos estudos sobre o tema, razão pela qual o objetivo do presente estudo foi propor algumas reflexões e introduzir problemáticas nesse campo de estudo.

O presente trabalho destacou as tecnologias de Sistemas Especialistas dentro do campo de pesquisa da IAM sob a perspectiva de analisar questões de Propriedade Intelectual, Responsabilidade e demais fatores sobre a práticas e componentes desses sistemas envolvendo tecnologias de Inteligência Artificial e Big Data.

Foi observado que, mesmo a IAM sendo um campo de estudo existente há mais de 40 anos, os últimos desenvolvimentos em tecnologias de Inteligência Artificial e processamento de dados trouxeram novos desafios à regulação e utilização de tais tecnologias e que ainda não foram abordados de maneira expressiva pela doutrina.

No que se refere à privacidade, questionou-se a respeito da privacidade dos dados utilizados por sistemas especialistas, destacando a multiplicidade de fontes de informações que alimentam os Sistemas Especialistas, algumas de caráter público e

\footnotetext{
${ }^{33}$ Existem várias as formas sob as quais podem tentar ser reguladas as questões de responsabilidade por danos causados por agentes de inteligência artificial. Uma delas seria a aplicação do princípio geral do Art. 12 da Convenção das Nações Unidos sobre o Uso das Comunicações Eletrônicas em Contratos Internacionais, onde a pessoa jurídica ou física para qual um determinado programa fosse programado, seria responsável por qualquer mensagem gerada por aquela máquina, reforçando assim a interpretação de que uma ferramenta não teria vontade própria e que o responsável por essa ferramenta seria, portanto, também responsável pelos resultados causados por esta (ČERKAA; GRIGIENĖA; SIRBIKYTĖB, 2015 p. 384).
} 
outras que levantam questionamento acerca da possibilidade de utilização de certos dados. Para permitir o uso de determinados dados para desenvolvimento futuros, foram apresentados também mecanismos de 'desidentificação' de dados e algumas consequências de seus usos.

No que se refere à questão ética, Guarizi e Oliveira (2014, p. 36) entendem que os sistemas computacionais dotados de Inteligência Artificial para a área da saúde podem acabar substituindo o médico especialista por conta da sua extensa base de conhecimento e capacidade avançada de tomada de decisões baseadas em uma vastidão de dados. Merece ainda atenção o entendimento de Luxton (2014), que destaca as preocupações acerca das aplicações de tecnologias de Inteligência Artificial em determinados campos da saúde, como seria o caso da saúde mental, onde o elemento humano, a sensibilidade e empatia, que não podem ser reproduzidas ainda por sistemas de IA, são essenciais para o devido processo terapêutico.

No que se refere à Propriedade Intelectual, foram traduzidos alguns comentários no que se refere a questões relacionadas aos conceitos fundamentais do direito autoral, dando destaque às problemáticas envolvendo o papel dos sistemas de Inteligência Artificial e do Big Data nas bases de dados. As questões de Propriedade Intelectual foram apresentadas com o objetivo de problematizar a questão, principalmente acerca da adequação da legislação atual a essas novas tecnologias.

Nesse sentido, a primeira conclusão do presente trabalho foi que não existem ainda muitos estudos que tratam das problemáticas aqui expostas, principalmente aquelas referentes à Propriedade Intelectual e os impactos das novas tecnologias aqui mencionadas no campo do Direito da Propriedade Intelectual.

Outra conclusão seria ratificar a importância acerca da reflexão sobre a regulação dessas novas tecnologias envolvendo bases de dados e tecnologias de Inteligência Artificial ao se verificar o aumento do uso de sistemas dotados de Inteligência Artificial e a sua natureza dinâmica e constantemente mutável, seja pela legislação atual, seja pela proposição de novas normas.

A terceira conclusão é acerca da impossibilidade de se aplicar os entendimentos acerca da utilização de tecnologias de Inteligência Artificial para todos os campos da saúde e da mesma maneira em todos os casos. A autonomia, inclusive decisória, de tais tecnologias poderiam ser maiores ou menores em cada campo da saúde, relacionando-se 
ISSN ELETRÔNICO 2316-8080

A Inteligência Artificial e o Big Data no Setor da Saúde: Os Sistemas Especialistas e o Direito.

com outros aspectos, tais como a importância do fator humano em uma determinada prática.

Por fim, importante se faz também uma maior reflexão acerca do tratamento dos dados e imagens utilizadas por sistemas especialistas de diagnósticos e questões de privacidade, sob a perspectiva de se privilegiar a pesquisa nesse setor através do uso de tais dados, utilizando mecanismos e tecnologias que impediriam a identificação de um determinado paciente sempre que necessário.

\section{REFERÊNCIAS}

ALBAGLI, Sarita; APPEL, Andre Luiz; MACIEL, Maria Lucia. E-Science e Ciência Aberta: Questões em Debate. XIV Encontro Nacional de Pesquisa em Ciência da Informação (ENANCIB 2013).

BERMAN, Jules J. Confidentiality issues for medical data miners. Artificial Intelligence in Medicine. 26. 2002. Pp. 25-36.

BRASIL. Constituição da República Federativa do Brasil de 1988. Brasília, DF.

BRASIL. Lei No 9.609 , de 19 de Fevereiro de 1998. Dispõe sobre a proteção da propriedade intelectual de programa de computador, sua comercialização no País, e dá outras providências. Brasília, DF.

BRASIL. Lei $N^{\circ} 9.610$, de 19 de Fevereiro de 1998. Altera, atualiza e consolida a legislação sobre direitos autorais e dá outras providências. Brasília, DF.

BRASIL. Lei $\mathbf{N}^{\circ}$ 10.406, de 10 de janeiro de 2002. Institui o Código civil. Brasília, DF.

BRETERNITZ, Vivaldo José; SILVA, Leandro Augusto. Big Data: Um novo Conceito gerando oportunidades e desafios. Revista RETC - Edição 13a . Outubro de 2013. P.106.

BRYNJOLFSSON, E., MCAFEE, A. The Second Machine age: Work, Progress, and Prosperity in a Time of Brilliant Technologies, W. W. Norton \& Company, New York, NY. 2014.

CARLONI, Giovanna Louise Bodin de Saint-Ange Comnène. Privacidade e Inovação na Era do Big Data. Trabalho de Conclusão de Curso apresentado à FGV Direito Rio como requisito parcial para obtenção do grau de bacharel em Direito. Rio de Janeiro. Junho de 2013.

CASTRO JÚNIOR, Marco Aurélio de. Direito e Pós-Humanidade. Quando os Robôs serão sujeitos de Direito. Curitiba. Editora Juruá. 2013.

ČERKAA, Paulius; GRIGIENĖA, Jurgita; SIRBIKYTĖB, Gintarè. Liability for damages caused by artificial intelligence. Computer Law \& Security Review. Vol 31. No.3. Junho de 2015. Pp. 376-389

DAVIES, Colin R. An evolutionary step in intellectual property rights - Artificial Intelligence and Intellectual Property. Computer Law \& Security Review. Vol 27. 2011. Pp. 601-619. 
FERNANDES, A.P.S. Sistema Especialista Difuso de Apoio ao Aprendizado do Traumatismo Dento-Alveolar Utilizando Recursos Multimídia. Dissertação de Mestrado. Curso de Pós-graduação em Engenharia de Produção e Sistemas. Universidade Federal de Santa Catarina, Florianópolis, 1997.

GONÇALVES, Victor Hugo Pereira. Big Data, Propriedade Intelectual e a segurança de informação: o cidadão comum na nuvem de dados. Migalhas de Peso. 22 de maio de 2014. Acesso em 2 mar 2016.

GUARIZI, Débora Deflim; OLIVEIRA, Eliane Vendramini. Estudo da Inteligência Artificial aplicada na área da saúde. Colloquium Exactarum, vol. 6, n. especial, JulDez, 2014, p. 26-37.

HENGSTLER, M.; ENKEL, E.; DUELLI, S. Applied artificial intelligence and trust the case of autonomous vehicles and medical assistance devices. Technological Forecasting \& Social Change, 105. 2016. Pp. 105-120.

JASZI, Peter. Toward a theory of copyright: The metamorfoses of "Autorship". Duke Law Journal, Vol. 1991, n. 2 (Apr., 1991), pp. 455-502.

KEMP, Richard. Legal Aspects of managing Big Data. Computer Law \& Security Review. 30. 2014. Pp. 482-491.

LUXTON, David D. Recommendations for the ethical use and design of artificial inteligente care providers. Artificial Intelligence in Medicine 62 (2014). Pp. 1-10

MCMILLAN, R.; DWOSKIN, E. IBM Crafts a role for Artificial Intelligence in Medicine. The Wall Street Journal. Aug 11, 2015. Disponível em http://www.wsj.com/articles/ibm-crafts-a-role-for-artificial-intelligence-in-medicine1439265840. Acesso em 16 Jul 2016

RUSSELL, S.J., NORVIG, P., 1995. Artificial Intelligence: A Modern Approach. Prentice Hall,. Englewood Cliffs, New Jersey.

SCHIRRU, L. A Inteligência Artificial e o Direito Autoral: primeiras reflexões e problematizações. Boletim do GEDAI, Edição de Agosto, 2016. Disponível em: http://www.gedai.com.br/?q=pt-br/boletins/boletim-gedai-agosto-

2016/intelig\%C3\%AAncia-artificial-e-o-direito-autoral-primeiras-reflex\%C3\%B5es-e.

SCHIRRU, L. Inteligência Artificial e o Direito: Questões de propriedade intelectual e responsabilidade aplicadas a agentes de inteligência artificial e robótica. Anais do IX Congresso de Direito de Autor e Interesse Público. Curitiba. 2015. P.57

TURING, Alan M. Computing Machinery and Intelligence. Mind. Vol. 49. 1950. Pp. 433-460. p. 433

VACCA, Ryan. Intellectual Property and Public Health - A White Paper. Akron Intelllectual Propery Journal (Forthcoming 2013). The University of Akron School of Law. Legal Studies Research Paper Series. 2013. Disponível em http://ssrn.com/abstract=2259089.

WACHOWICZ, Marcos. A Proteção Jurídica Das Bases De Dados Em Face Da Revolução Da Tecnologia Da Informação. P.14. Disponível em: http://www.gedai.com.br/sites/default/files/arquivos/artigo-base-dados-marcoswachowicz.pdf.

WESTPHAL, J. T.; Modelagem Difusa de um Sistema Especialista Médico: Avaliação dos Fatores de Internação em Crianças Queimadas. 2003. 123f. 
ISSN ELETRÔNICO 2316-8080

A Inteligência Artificial e o Big Data no Setor da Saúde: Os Sistemas Especialistas e o Direito.

Dissertação (Mestrado) - Programa de Pós - Graduação em Ciência da Computação, Universidade federal de Santa Catarina, Florianópolis, 2003.

WIDMAN, Lawrence, E. Sistemas Especialistas em Medicina. Informática Médica. Vol. $1 . \quad$ N. $5 . \quad$ Set/Out $1998 . \quad$ Disponível em http://www.informaticamedica.org.br/informaticamedica/n0105/widman.htm. Acesso em 15 jul 2016.

YANG C. C, VELTRI P, Intelligent healthcare informatics in big data era, Artificial Intelligence in Medicine (2015), http://dx.doi.org/10.1016/j.artmed.2015.08.002.

RECEBIDO 25/08/2016

APROVADO 25/09/2016

PUBLICADO 31/10/2016 